\title{
Laboreal
}

Volume $10 \mathrm{~N}^{\circ} 2$ | 2014

Análise ergonómica do trabalho e formação - Parte II

\section{Introducción a la colección temática "Análisis ergonómico del trabajo y formación": el análisis ergonómico del trabajo y de la formación para un desarrollo sustentable - parte II}

Introdução ao dossiê «Análise ergonómica do trabalho e formação»: A análise ergonómica do trabalho e da formação para um desenvolvimento durável - parte II

Introduction au dossier thématique "Analyse ergonomique du travail et formation ": L'analyse ergonomique du travail et de la formation pour un développement durable - part II

Introduction to the thematic dossier "Ergonomic work analysis and training»: The ergonomic work analysis and training for a lasting development - part II

\section{Céline Chatigny}

\section{OpenEdition}

Journals

Edición electrónica

URL: http://journals.openedition.org/laboreal/4685

DOI: $10.4000 /$ laboreal. 4685

ISSN: 1646-5237

Editor

Universidade do Porto

Referencia electrónica

Céline Chatigny, «Introducción a la colección temática "Análisis ergonómico del trabajo y formación": el análisis ergonómico del trabajo y de la formación para un desarrollo sustentable - parte II », Laboreal [En línea], Volume 10 N²$^{\circ}$ | 2014, Publicado el 01 diciembre 2014, consultado el 24 septiembre 2020. URL : http://journals.openedition.org/laboreal/4685 ; DOI : https://doi.org/10.4000/ laboreal.4685

Este documento fue generado automáticamente el 24 septiembre 2020.

Laboreal está licenciado com uma Licença Creative Commons - Atribuição-NãoComercial 4.0 Internacional. 


\section{Introducción a la colección temática "Análisis ergonómico del trabajo y formación": el análisis ergonómico del trabajo y de la formación para un desarrollo sustentable - parte II}

Introdução ao dossiê «Análise ergonómica do trabalho e formação»: A análise ergonómica do trabalho e da formação para um desenvolvimento durável - parte II

Introduction au dossier thématique « Analyse ergonomique du travail et formation »: L'analyse ergonomique du travail et de la formation pour un développement durable - part II

Introduction to the thematic dossier «Ergonomic work analysis and training»: The ergonomic work analysis and training for a lasting development - part II

\section{Céline Chatigny}

\section{NOTA DEL EDITOR}

http://dx.doi.org/10.15667/laborealx0214cc

Presentamos la segunda parte de la colección temática sobre el análisis ergonómico del trabajo y la formación. Para los lectores que aún no han tenido la oportunidad de leer la primera parte de dicha colección en el número anterior, recordamos que la originalidad de este proyecto resulta de la publicación del conjunto de los textos del simposio " Ergonomics work analysis and training (EWAT)». Este simposio se llevó a cabo durante el 18o congreso de la Asociación Internacional de Ergonomía (2012, Recife, Brasil) con el tema "Designing a sustainable future". Reunió a investigadores y consultores de 
diversos países (Bélgica, Brasil, Canadá, Francia, Portugal y Suiza) y se realizó en la continuidad de otros simposios EWAT, realizados desde 1991, sobre la contribución del análisis ergonómico para las situaciones de formación y de aprendizaje [1]. Los textos del simposio se publicaron en las revistas Laboreal (en dos números) y PISTES (todos los textos serán publicados en breve: http://pistes.revues.org/).

2 La contribución de la ergonomía para los temas de la formación se construyó de forma coherente con el objetivo de la disciplina, es decir, la adaptación del trabajo a las capacidades humanas. De modo que el diseño de dispositivos de formación debería favorecer el desarrollo de las condiciones de aprendizaje y de trabajo para permitir el desarrollo de las competencias y de la salud. Las contribuciones se incluyen, pues, en una perspectiva sistémica y constructivista.

3 Los tres textos de esta colección temática se encuadran en tres de los cuatro temas que fueron tratados en el simposio [2].

El primer artículo, de Sandrine Cortessis, está relacionado con la temática 1 del simposio, "Transmisión y construcción de saberes en contexto de trabajo". El contexto del estudio es el de la implementación de un dispositivo inédito de Validación de la Experiencia Adquirida (VAE) en la enseñanza, resultante de una nueva legislación en Suiza. Con el fin de formar los evaluadores (que son ellos mismos profesores), se concretó un dispositivo colectivo de análisis de las prácticas de evaluación por el comité de evaluadores.

5 El segundo artículo de Claire Tourmen, Annie Leroux, et Sylvie Beney se refiere a los primeros aprendizajes en el trabajo y se incluye en la temática 2 del simposio, "Análisis de la actividad de personas en formación y formadores y de sus enfoques pedagógicos". El estudio se realizó en Francia, en dos organizaciones, con Asistentes de Servicio Social (ASS) y personal en prácticas, en un contexto de reorganización de las trayectorias de prácticas, en el seguimiento de una reforma que busca instaurar trayectorias cualificantes.

El tercero artículo, de Céline Chatigny y de Jessica Riel, coincide con el tema 4 "Formación y aprendizaje para poblaciones específicas", sesión que se organizó con el Comité técnico «Gender and Work (Género y Trabajo)» en el ámbito del congreso del IEA. En este texto, el análisis de la actividad se refiere a la situación de los estudiantes en contexto de aprendizaje en talleres-escuelas en Quebec. El análisis ergonómico se conjugó aquí con un análisis diferenciado según el sexo/género. Así se pone de manifiesto un tratamiento diferenciado de la salud y seguridad de los alumnos en contextos tradicionalmente femeninos y tradicionalmente masculinos, tanto en saberes definidos en el plan de estudios, como en las actividades y en las condiciones de aprendizaje en los talleres.

7 Estas tres contribuciones se relacionan con el medio escolar: una se refiere a la situación de los profesores-evaluadores y las otras dos a situaciones de estudiantes que están aprendiendo un oficio o una profesión. Esta constatación es interesante pues la ergonomía de la actividad del trabajo, basada en entrevistas y observaciones en contexto real, se ha dedicado de forma más intensa al medio escolar recientemente (Messing et Seifert, 2001; Rogalsky, 2004; Cau-Bareille, 2009). Quizá se trate de un avance en el medio escolar, pero solo lo podremos evaluar mejor en los próximos años. Y esto puede estar relacionado con otra constatación que es la de un acercamiento en los últimos años (o, al menos, de curiosidades crecientes) entre la ergonomía de la 
actividad y la didáctica profesional. Notemos que dos de las contribuciones se apoyan en la didáctica profesional y que ésta se asocia generalmente a los medios de la formación sin tratar de influenciar directamente en las condiciones de trabajo. Aunque en estos estudios no se efectuaron observaciones in situ, se pudo influir en ciertas condiciones de aprendizaje.

La tercera contribución, que analizó las observaciones de las actividades de los estudiantes en contexto de aprendizaje, permitió identificar las condiciones problemáticas del aprendizaje y del trabajo, a pesar de un número de observaciones menos importante que el previsto.

Cabe preguntarse si el medio escolar es más limitador que otros medios profesionales para la realización de observaciones. En particular porque el profesor/tutor progresa habitualmente solo con las personas en formación/prácticas, lo que confiere al observador una posición de intrusión aún más marcada en lo cotidiano de los actores. Se puede pensar igualmente que el contexto de intensificación del trabajo, que afecta también el medio escolar, hace el trabajo del ergónomo más difícil. En cualquier caso, es conveniente cuestionar el estatuto y las contribuciones de las observaciones que permiten enriquecer los conocimientos sobre el trabajo y que normalmente deberían recalcar las repercusiones en las condiciones de trabajo/aprendizaje.

Son varias las cuestiones en discusión en el grupo de los ergónomos y de los investigadores en el campo de la didáctica profesional que se juntan en los simposios EWAT, basándose en marcos teóricos y metodológicos diversos y también en perspectivas diferentes. ¿Cuales son, entonces, los puntos de convergencia y de divergencia de estos enfoques y de los trabajos que resultan de ellos? ¿Cuales son las asociaciones posibles?

11 El artículo de Vidal-Gomel, Delgoulet et Geffroy (2014), publicado en el número anterior de la revista, ofrece algunas pistas al aprehender conjuntamente los retos relacionados con las opciones de organización de las intervenciones de urgencia de los bomberos, la dimensión ampliamente colectiva del manejo de los vehículos de emergencia y el contenido de la formación de los conductores de estos vehículos. El reciente trabajo de Mayen (2014), en el cual se propone una síntesis de los marcos de la didáctica profesional y se identifican algunos limites de los estudios que se refieren a este enfoque, parece también ser prometedor de nuevas perspectivas de investigación. Tantas cuestiones que podrían originar un nuevo simposio.

12 Como un todo, estas tres contribuciones atestiguan la relevancia del análisis del trabajo para el desarrollo de dispositivos de formación y la importancia de influir en las condiciones de aprendizaje y de trabajo. Esperamos que estos textos estimulen otros investigadores y consultores a aprovechar y a desarrollar los conocimientos aportados por la «ergonomía en el campo de la formación».

13 Para terminar, agradecemos de todo el corazón a la revista Laboreal su apoyo en la concepción y realización del proyecto. Agradecemos también a los autores que han aceptado el reto de una doble publicación en Laboreal y en PISTES.

¡Muy buena lectura!

15 Céline Chatigny en representación de los colegas del comité organizador del simposio: Dominique Cau-Bareille, Catherine Delgoulet, Marie Laberge, Sylvie Ouellet y Marta Santos. 


\section{BIBLIOGRAFÍA}

Cau-Bareille, D. (2009). Vécu de travail et santé des enseignants en fin de carrière : une approche ergonomique. Rapport de recherche 56. Centre d'études de l'emploi.

Mayen, P. (2014). Lever quelques embarras et incertitudes de méthodes en didactique professionnelle. Travail et Apprentissage, 13, 118-138.

Messing, K., \& Seifert, A. M. (2001). «On est là toutes seules». Contraintes et stratégies des femmes en contrat à durée déterminé dans l'enseignement des adultes. Travailler, 7, 1, 147-166.

Rogalski, J. (2004). Y a-t-il un pilote dans la classe ? Une analyse de l'activité de l'enseignant comme gestion d'un environnement dynamique ouvert. Recherches en didactique des mathématiques, 23,3, 343-388.

\section{NOTAS}

1. Las contribuciones de la ergonomía para los temas de la formación se valorizaron en los congresos anteriores y pueden ser consultadas en las publicaciones siguientes: Número especial: Work, 42, 1, 2012: (http://iospress.metapress.com/content/q66420837m77) Lacomblez, M., Bellemare, M., Chatigny, C., Delgoulet, C., Re, A., Trudel, L., and Vasconcelos, R. (2007). Ergonomic analysis of work activity and training: basic paradigm, evolutions and challenges. In, R. Pikaar, E. Koningsveld, and P. Settels, (eds.), Meeting diversity in ergonomics (129-142), Amsterdam and Boston: Elsevier. (https://www.elsevier.com/books/meetingdiversity-in-ergonomics/pikaar/978-0-08-045373-6).

Número especial: PISTES (http://www.pistes.uqam.ca), 6, 2, 2004.

Número especial: Relations industrielles/Industrial Relations, 56, 3, 2001.

Número especial: Safety Science, 23, 2/3, 1996.

Número especial: L'ergonome, le formateur et le travail. Education Permanente, 124, 1995.

2. El tema 3 no está representado en esta segunda parte de la colección temática: análisis, evaluación y transferencia de las prácticas de formación.

\section{AUTOR}

\section{CÉLINE CHATIGNY}

Université du Québec à Montréal, Département d'éducation et formation spécialisées, C.P.8888, Succ. Centre-ville Montréal (Québec), Canada, H3C 3P8

chatigny.celine@uqam.ca 